\title{
Damage Characteristics of Silicone Rubber caused by Abrading The Edge of PPC Papers cut with $\mathrm{CO}_{2}$ Laser
}

\author{
Moriaki Wakaki $^{1)}$, Naohiro Takahashi ${ }^{2}$, Norihiko Sawaki ${ }^{2)}$ and \\ Shuichi Maeda ${ }^{1), 2)}$ \\ ${ }^{1)}$ Department of Optical and Imaging Science \& Technology, Faculty of Engineering, Tokai University ${ }^{* 1}$ \\ ${ }^{2)}$ Course of Electrical and Electronic Engineering, Graduate School of Engineering, Tokai University ${ }^{* 1}$
}

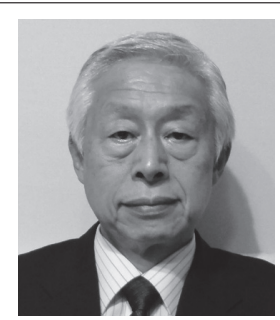

Moriaki Wakaki

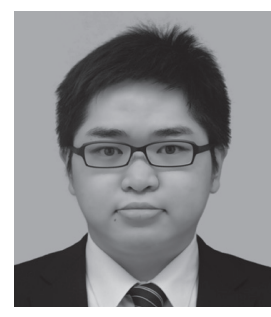

Norihiko Sawaki

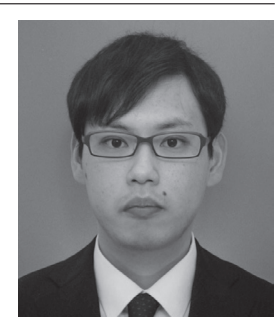

Naohiro Takahashi

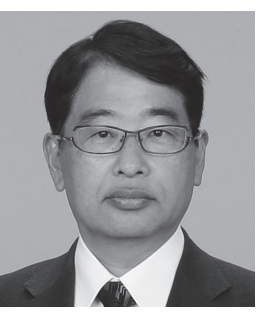

Shuichi Maeda

\begin{abstract}
We propose to use a carbon dioxide laser to cut the paper which provides two benefits. The blade of the cutting system will not wear without a mechanical cutter and the cut surface can be smoothened by choosing the optimal irradiation of the laser which prevent accidental cutting of the human skin. In the experiment to demonstrate these benefits, a $\mathrm{CO}_{2}$ laser, a condenser lens, a rotating table, and a silicone rubber that simulates the human skin were used. The thresholds that can cut all the papers used in the experiment with a $\mathrm{CO}_{2}$ laser was obtained. Simulation experiments using a silicone rubber to characterize the damage caused by the abrasion of the paper edge suggested that the paper edge cut by use of a $\mathrm{CO}_{2}$ laser was gentler to the skin than the edge cut by use of scissors.
\end{abstract}

Keywords : Paper cutting system, $\mathrm{CO}_{2}$ laser, Smooth surface of the paper edge, Gentle to human skin

\section{Background}

Roll papers are generally cut at a proper width by using a slitting at the finishing process in the paper mill. However, after cutting many papers, the blade of the

\footnotetext{
${ }^{* 1} 4-1-1$ Kitakaname, Hiratsuka-shi, Kanagawa 259-1292, Japan
}

slitter becomes wear and the replacement of the blade is the problem of labor hour and cost. Routine replacement of the wear down slitter might be avoided by using a $\mathrm{CO}_{2}$ laser (lasing wavelength ; $10.6 \mu \mathrm{m}$ ) to cut papers. The $\mathrm{CO}_{2}$ laser is utilized in the industry for drilling, cutting, welding, and thermal treatment of metals ${ }^{1)-4)}$, but the reflectivity of the $\mathrm{CO}_{2}$ laser light to metals is high and non-metals are better for processing by the 
Table 1 Physical properties of various PPC papers

\begin{tabular}{|c|c|c|c|c|c|}
\hline Model & HQ-500 & G70 & J & SG & V-Paper \\
\hline Maker / Supplier & NP TRADING Co., Ltd & Fuji Xerox Co., Ltd & Fuji Xerox Co., Ltd & Fuji Xerox Co., Ltd & Fuji Xerox Co., Ltd \\
\hline Weight (g/m²) & 64.7 & 66.1 & 78.5 & 63.7 & 65.1 \\
\hline Thickness ( $\mu \mathrm{m})$ & 90 & 92 & 98 & 89 & 86 \\
\hline Density (g/cm $\left.{ }^{3}\right)$ & 0.72 & 0.72 & 0.80 & 0.72 & 0.75 \\
\hline $\begin{array}{c}\text { Smoothness (sec) } \\
\text { front face }\end{array}$ & 28.0 & 30.7 & 79.0 & 42.7 & 46 \\
\hline $\begin{array}{c}\text { Smoothness (sec) } \\
\text { rear face }\end{array}$ & 19.7 & 31.0 & 89.0 & 36.7 & 34 \\
\hline Air permeability (sec) & 10.9 & 13.4 & 14.9 & 8.3 & 11.8 \\
\hline ISO brightness (\%) & 81.8 & 69.0 & 86.5 & 82.1 & 81.2 \\
\hline Specular gloss 75 (\%) & 4.0 & 6.6 & 7.7 & 6.3 & 6.2 \\
\hline
\end{tabular}

laser than metals due to their absorption efficiencies ${ }^{5)-7)}$.

There is another large merit to use a $\mathrm{CO}_{2}$ laser in addition to the above mentioned merit on cutting papers. We often injure the skin of a finger on rubbing the finger carelessly by the edge of a commercially available PPC (Plain Paper Copier) paper. Fiber of a paper is jagged and the edge of the paper cut by the mechanical cutter is notched like a saw blade. For that reason, the cut portion of the wound is damaged largely. Especially, a fingertip is the portion of excessive sensibility and we feel a sharp pain. Such wounds are often occurred on the use of a copy machine in the office or field. A Guillotine cutter is usually used to cut PPC papers. It is considered the edge profile formed by the process tends to injure the skin. We expect to solve the problem by making a paper edge profile gentler for the human skin.

The purpose of this research is classified into following two studies.

(1) The first study is to obtain the threshold experimentally to offer a good cut edge for various papers by the laser cutting without burn marks in order to get the effective laser cutting condition.

(2) Second study is to obtain a paper edge profile gentler for the human skin. Commercially supplied papers, typically PPC papers, well used for copy machines are cut using a $\mathrm{CO}_{2}$ laser with various conditions. A silicone rubber sheet which is often used as an artificial skin or a robotic skin ${ }^{8)}$ is abraded by pressing and rubbing the cut paper edge at various moving speeds. The abraded damages on the silicone rubber sheet are observed by the scanning electron microscope (SEM) and we find out the laser irradiation condition to cut the paper which induces the minimum damage to the silicone rubber sheet.

\section{Experimental method}

\subsection{Materials for research}

Various type of PPC papers were studied and the physical properties of these papers are summarized on Table 1.

\subsection{Experimental instruments}

Silicone rubber sheets (Wacom silicone rubber sheet; Wacom Manufacturing Co., Ltd.) were used as a skin model ${ }^{8)}$. A RF excited sealing type $\mathrm{CO}_{2}$ laser (Edinburgh Instrument) with the output power of $5.5 \mathrm{~W}$ and the wavelength of $10.6 \mu \mathrm{m}$ was used. The laser emits a linear polarized beam and the beam intensity was controlled by adjusting the rotation angle of the infrared polarizer set outside of the laser. A ZnSe lens with a focal length of $8 \mathrm{~cm}$ was used to concentrate the laser beam. Various kind of paper cutting edges were obtained by irradiating the laser beam to the paper attached to the rotating disk. The cutting speed of the paper was controlled by changing the rotation speed of the disk. The arrangement of the experimental setup is illustrated in Fig. 1. The rotating speed of the disk can be controlled at 7 steps. At the experiment, the laser is fixed and the paper is moving by the rotation. This offers same situation as the irradiation of the moving laser beam to the fixed paper. Then, the expression, such as "irradiation of the moving laser beam to the fixed paper" is often used hereafter.

The simulation experiments corresponding to the 


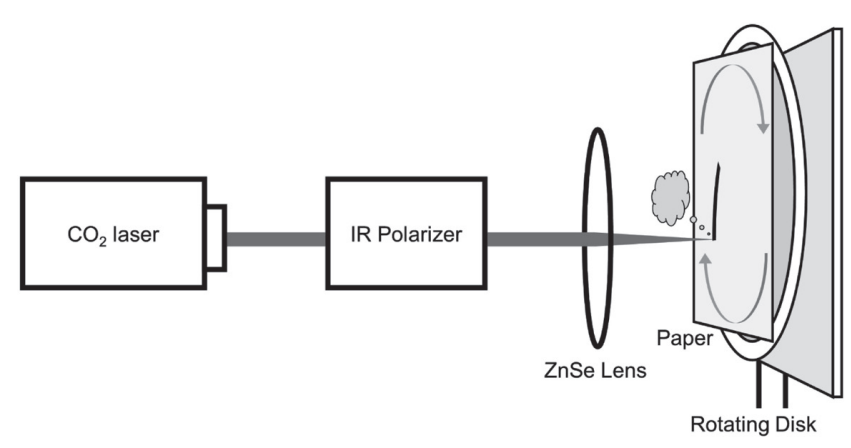

Fig. 1 Schematic diagram for cutting PPC paper at various scanning speeds using $\mathrm{CO}_{2}$ laser (lasing wavelength ; $10.6 \mu \mathrm{m}$ )

abraded damage of the human skin were carried out by using the rotating disk. The abraded damages on the silicone rubber sheets caused by pressing the moving edge of the PPC paper cut by the laser or the scissors to the circular disk shape were observed by the Scanning Electron Microscope (SEM VE-8800 ; KEYENCE Co., Ltd.).

\subsection{Experimental procedures}

Following three cases were studied in this research.

(1) A paper was set on the rotating disk at a distance of $8 \mathrm{~cm}$ from the $\mathrm{ZnSe}$ focusing lens and was cut by the laser beam of $2 \mathrm{~W}$ output at various scanning speeds. The scanning speed was changed between 5 and $25 \mathrm{~mm} / \mathrm{sec}$ with the step of $5 \mathrm{~mm} / \mathrm{sec}$. The edges of PPC papers cut by the $\mathrm{CO}_{2}$ laser were observed by the SEM. The untreated edge of a commercial PPC paper was also observed and compared with the laser cut edge by the SEM.

(2) The PPC paper was cut by the laser at the optimum scanning speed of $15.7 \mathrm{~mm} / \mathrm{sec}$ to a circular disk with the diameter of $30 \mathrm{~mm}$. The abraded damage of a silicone rubber sheet pressed by the rotating cut edge of the PPC paper at the speed of $\mathrm{v}=360 \mathrm{~mm} / \mathrm{sec}$ was observed by the SEM. The experimental setup is shown in Fig. 2. The distance between the edge of the PPC paper and the silicone rubber sheet was decreased and the contact point was set as $0 \mu \mathrm{m}$ (starting point). The edge of the paper was pressed into the silicone rubber sheet from the starting point to some insertion length lp with the step of $50 \mu \mathrm{m}$.

(3) The abraded damage depth ld induced on the silicone rubber sheet was observed by the SEM for different moving speeds of the paper at the fixed insertion length $\mathrm{lp}$. The abraded damage of the silicone rubber sheet by the edge of the PPC paper cut by scissors was also characterized for comparison.

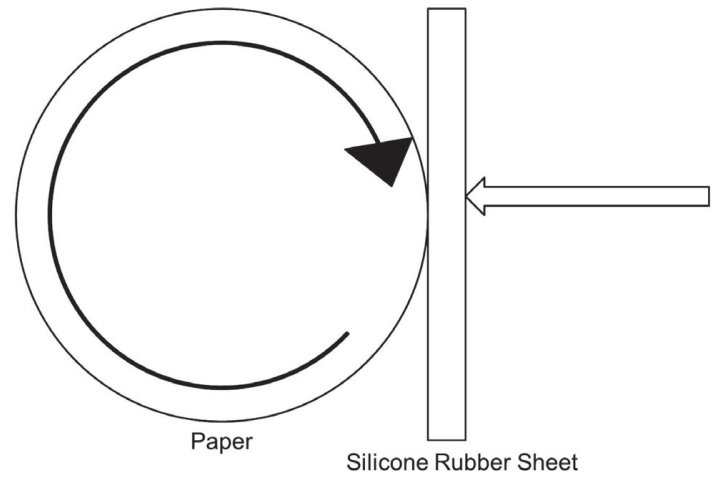

Fig. 2 Experimental setup to abrade silicone rubber sheet by pressing rotating circular paper cut by $\mathrm{CO}_{2}$ laser or scissors

\section{Experimental results and discussions}

3.1 Cutting speed dependences of various PPC papers by $\mathrm{CO}_{2}$ laser

The relations between the scanning speed of the laser and cut results of 5 kind of papers are summarized on Table 2 at the condition of the laser power of $2 \mathrm{~W}$ and the paper diameter of $40 \mathrm{~mm}$ cut to the disk shape. On the Table 2, \lceil\rfloor$,\lceil\triangle\rfloor$ and $\lceil\times\rfloor$ correspond to complete cutting, partial cutting and no cutting, respectively.

The focused minimum diameter $\mathrm{d}$ [mm] is given by the following formula (1) assuming an aplanatic lens, where $\mathrm{f}[\mathrm{mm}], \lambda[\mathrm{mm}]$ and $\mathrm{D}[\mathrm{mm}]$ are the focal length of the conversing lens, the wavelength and the diameter of the incident Gaussian laser beam, respectively ${ }^{9), 10)}$.

$$
\mathrm{d}=4 \mathrm{f} \lambda / \pi \mathrm{D}
$$

In this study, the diameter $\mathrm{D}$ of the incident laser beam is estimated from the processed mark caused by the short time laser irradiation to the sample like thermal paper. The obtained incident beam diameter $\mathrm{D}$ was around $2 \mathrm{~mm}$. The minimum focused beam diameter $\mathrm{d}$ was derived as $0.54 \mathrm{~mm}$ from the relation (1). As a result, the power density per unit area $\left(\mathrm{cm}^{2}\right)$ is given as $873.3 \mathrm{~W} / \mathrm{cm}^{2}$. The power density given to the unit

Table 2 Relation between cut results of PPC papers and scanning speed of $\mathrm{CO}_{2}$ laser

\begin{tabular}{|c|c|c|c|c|c|}
\hline $\begin{array}{c}\text { Scanning speed of } \mathrm{CO}_{2} \text { laser } \\
(\mathrm{mm} / \mathrm{sec})\end{array}$ & $\mathrm{HQ}-500$ & G70 & J & SG & V-Paper \\
\hline 35.9 & $\times$ & $\times$ & $\times$ & $\times$ & $\times$ \\
\hline 31.4 & $\triangle$ & $\times$ & $\times$ & $\times$ & $\times$ \\
\hline 27.9 & $\triangle$ & $\times$ & $\times$ & $\times$ & $\times$ \\
\hline 25.1 & $\bigcirc$ & $\times$ & $\times$ & $\times$ & $\times$ \\
\hline 20.9 & $\bigcirc$ & $\times$ & $\times$ & $\triangle$ & $\bigcirc$ \\
\hline 16.7 & $\bigcirc$ & $\bigcirc$ & $\triangle$ & $\bigcirc$ & $\bigcirc$ \\
\hline 14.0 & $\bigcirc$ & $\bigcirc$ & $\bigcirc$ & $\bigcirc$ & $\bigcirc$ \\
\hline
\end{tabular}


volume of each PPC paper $\mathrm{Pv}\left(\mathrm{Wsec} / \mathrm{cm}^{3}\right)$ is given by dividing the incident power density by the scanning velocity of the laser beam.

The power densities incident into the PPC papers which correspond to the threshold to start cutting estimated by using the values on Table 2 are summarized on Table 3. The paper $\mathrm{HQ}^{-} 500$ has the lowest threshold $\mathrm{Pv}=382.2 \mathrm{Wsec} / \mathrm{cm}^{3}$ and the paper J requires more than $\mathrm{Pv}=660.6 \mathrm{Wsec} / \mathrm{cm}^{3}$ to start cutting.

The PPC paper HQ-500 was studied in the following experiments for its lowest cutting threshold. HQ-500 is

Table 3 Correlation between the scanning speed $\mathrm{v}$ of the laser beam irradiated to PPC paper and the threshold incident energy per unit volume $\mathrm{Pv}$ to start cutting of the paper

\begin{tabular}{|c|c|c|}
\hline Model & $\mathbf{v}(\mathrm{mm} / \mathrm{sec})$ & $\mathrm{Pv}\left(\mathrm{Wsec} / \mathrm{cm}^{3}\right)$ \\
\hline HQ-500 & 22.85 & 382.2 \\
\hline G70 & 16.76 & 521.1 \\
\hline J & 13.22 & 660.6 \\
\hline SG & 16.76 & 521.1 \\
\hline V-paper & 19.33 & 451.8 \\
\hline
\end{tabular}

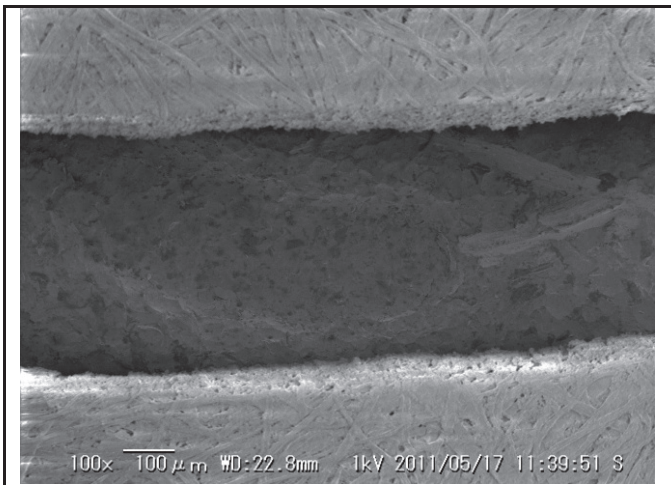

(a) v: $10.5 \mathrm{~mm} / \mathrm{sec}$

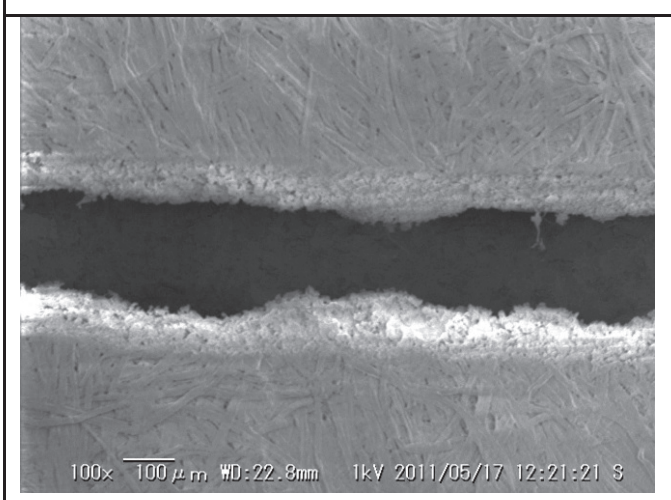

(c) v: $20.9 \mathrm{~mm} / \mathrm{sec}$ characterized by its density, gloss and smoothness compared with other papers. The low density of the paper gives the easy cutting, and the low gloss and smoothness give enhanced absorption of the incident light. For these reasons, it is estimated the paper has the lowest cutting threshold for the laser cutting.

3.2 Correlation between scanning speed of $\mathrm{CO}_{2}$ laser and the cut width of PPC paper

SEM images of the edges of a PPC paper (HQ-500) cut by the $\mathrm{CO}_{2}$ laser with the incident intensity of $2 \mathrm{~W}$ are shown in Fig. 3 for different scanning speeds of the laser beam. The cut width (width of the dissipated region by the irradiation of the laser beam) becomes smaller as the scanning speed becomes larger due to the decrease of the incident energy into the unit length. The large roughness of the cut edge is observed for the fast scanning speed due to the smaller incident energy per unit length. On the other hand, the cut width becomes larger and the roughness of the cut edge becomes smaller for the slower laser scanning speed. We selected as the optimum scanning speed of $15.7 \mathrm{~mm} / \mathrm{sec}$ considering these results of the roughness and the cut width of the paper edge. The enlarged SEM image of the cut edge of HQ-500 is shown in Fig. 4 cut with the scanning speed of $15.7 \mathrm{~mm} / \mathrm{sec}$. It is recognized the edge surface has a round shape compared with the untreated

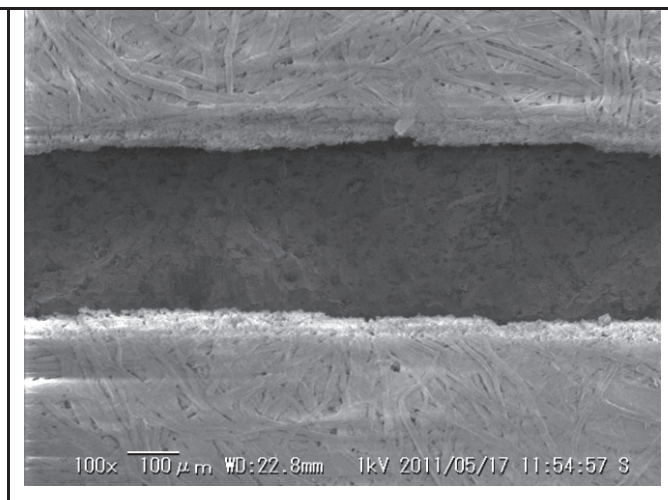

(b) v: $15.7 \mathrm{~mm} / \mathrm{sec}$

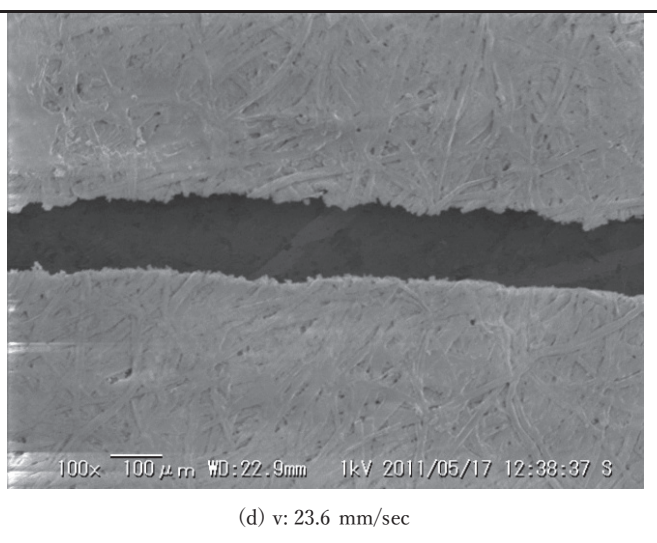

Fig. 3 SEM images of cut edges of PPC paper (HQ-500) cut at different laser scanning speeds $\mathrm{v}(\mathrm{mm} / \mathrm{sec})$ 


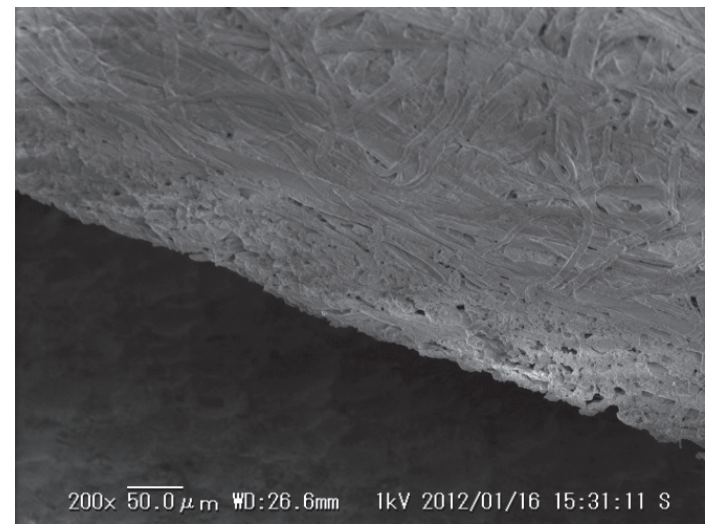

Fig. 4 Enlarged SEM image of the cut edge of PPC paper (HQ-500) cut at the laser scanning speed of $\mathrm{v}=15.7 \mathrm{~mm} / \mathrm{sec}$

edge of the commercial HQ-500 shown in Fig. 5.

The result that the cut width becomes narrower and the edge becomes rough as the laser scanning speed increases is estimated to be caused from the Gaussian distribution of the laser beam intensity profile. The incident energy per unit length becomes larger as the scanning speed of the laser beam becomes slower, and the enough energy to process the paper is offered even at the peripheral area of the Gaussian beam, which may

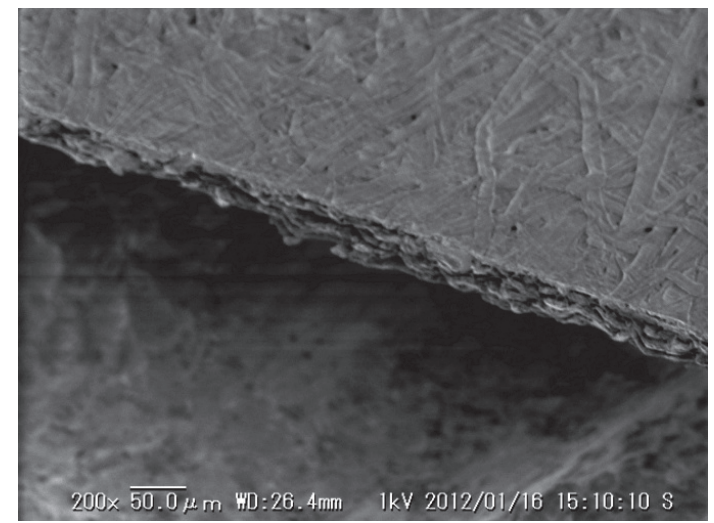

Fig. 5 Enlarged SEM image of the edge of commercial $\mathrm{PPC}$ paper (HQ-500)

cause a wide cut width. The reason that the high scanning speed causes a rough cut edge is estimated to be caused by the fluctuation of the laser power and the deviation of the paper positon from the focal point of the lens. As a result, these negative effects are tend to occur near the higher limit of the scanning speed to process the paper.

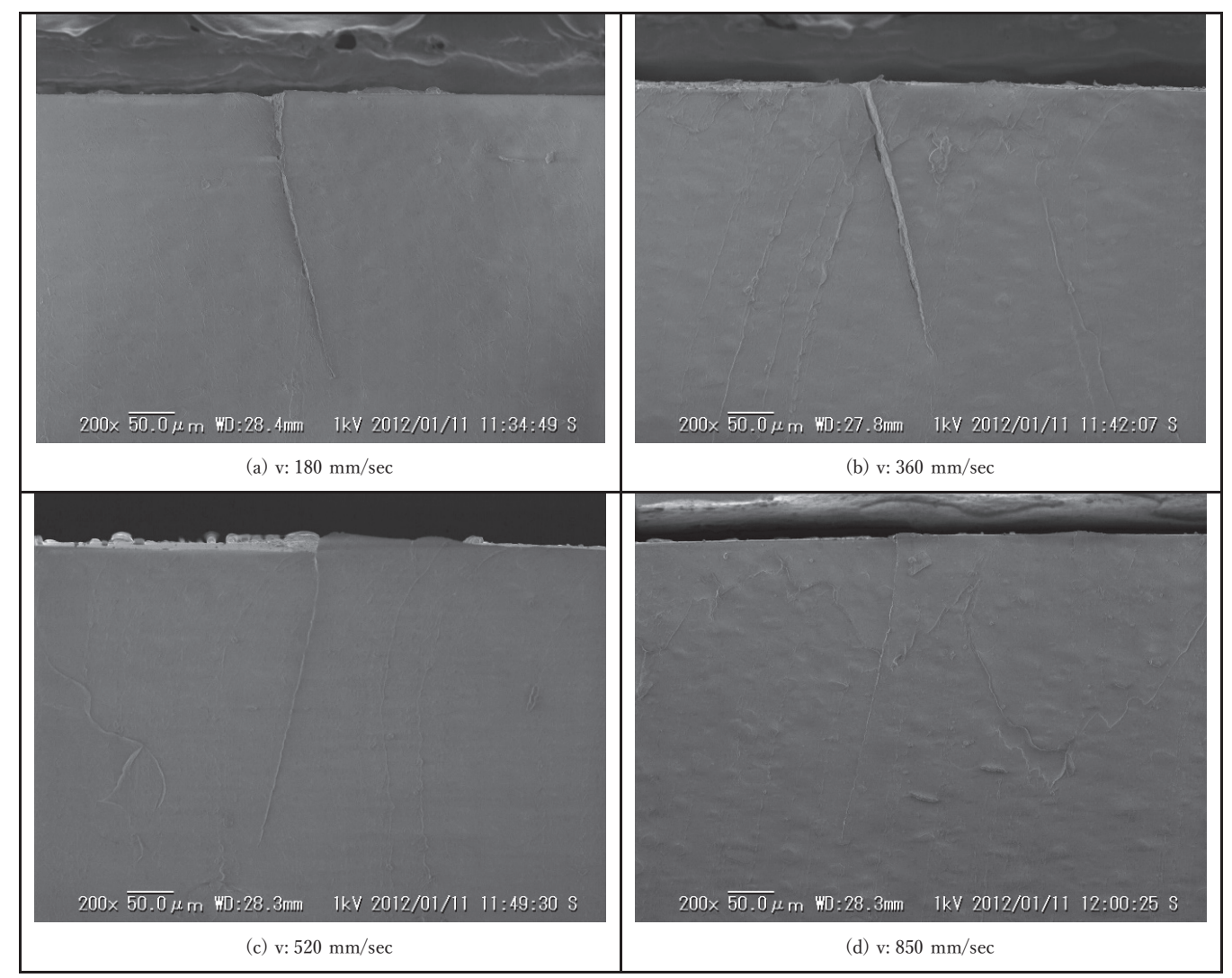

Fig. 6 Cross sectional SEM images of abraded damages on silicone rubbers caused by pressing the edge of the rotating paper with a constant insertion length at different moving speeds $\mathrm{v}(\mathrm{mm} / \mathrm{sec})$ 
3.3 Damage characteristics of silicone rubber sheet abraded by pressing moving PPC paper cut by $\mathrm{CO}_{2}$ laser : Effect of the moving speed of the paper

A PPC paper ( $\mathrm{HQ}-500)$ cut by the $\mathrm{CO}_{2}$ laser with the intensity of $2 \mathrm{~W}$ and the laser scanning speed of 15.7 $\mathrm{mm} / \mathrm{sec}$ was set on the rotating disk shown in Fig. 1. Cross sectional SEM images of abraded damages on silicone rubber sheets caused by pressing the edge of the rotating paper as shown in Fig. 2 with a constant insertion length $\mathrm{lp}$ at different moving speeds $\mathrm{v}(\mathrm{mm} /$ sec) are shown in Fig. 6. The depths ld of the abraded damage estimated from the SEM images Fig. 6 (a) (d) were almost $300 \mu \mathrm{m}$ in each case. As a result, the depth of the abraded damage caused on the silicone rubber sheet was found almost same for the change of the moving speed of the paper. For the reason, we selected the paper moving speed of $360 \mathrm{~mm} / \mathrm{sec}$ as a typical value in the following abraded damage experiments on silicone sheets. This moving speed almost corresponds to the rubbing speed when we injure our fingers by the abrasion of the paper edge carelessly.

3.4 Damage characteristics of silicone rubber sheet abraded by pressing moving PPC paper cut by $\mathrm{CO}_{2}$ laser : Effect of the pressing strength of the paper

Cross sectional SEM images of silicone rubber caused by pressing the edge of the rotating paper as shown in Fig. 2 at the moving speed of $\mathrm{v}=360 \mathrm{~mm} / \mathrm{sec}$ are shown in Fig. 7 for different insertion lengths $1 \mathrm{p}(\mu \mathrm{m})$. The insertion length lp shown in Fig. 7 is the pressing length of the paper from the contact point $l p=0$ and is considered to be proportional to the applied pressure. It is recognized from the SEM images Fig. 7 (a) (e) that the amount of the abraded damage on the silicone rubber becomes larger as the applied pressure of the rotating paper becomes stronger.

Correlation between the insertion length $\mathrm{lp}$ of the rotating paper $(\mathrm{v}=360 \mathrm{~mm} / \mathrm{sec})$ cut by the $\mathrm{CO}_{2}$ laser and the abraded damage depth ld on the silicone rubber sheet is shown in Fig. 8. The linear approximation in Fig. 8 (dotted line) is derived for the plots between $1 \mathrm{p}=150$ and $350 \mu \mathrm{m}$ using the least square method. The correlation coefficient of the linear approximation is 0.9971 and good linear approximation holds. The threshold of the insertion length lp which corresponds to the starting point of damage, was estimated $115.0 \mu \mathrm{m}$. As a result, a silicone rubber is not damaged for the lower abrading strength than the threshold lp by the edge of the paper cut by the laser.
3.5 Damage characteristics of silicone rubber sheet abraded by pressing moving PPC paper cut by scissors (cutting tool) : Effect of the pressing strength of the paper

The edges of the commercial papers are cut by using a Guillotine cutter. In this experiment, the abraded damage on the silicone sheet is characterized using a rotating circular paper and we used the edge of the paper cut by scissors which has almost same cut edge to the commercial paper. The abraded damage experiment using the PPC paper (H-Q500) cut by the scissors to a circular form was carried out using the same procedure in section 3.4. Cross sectional SEM images of the silicone rubber sheet caused by pressing the edge of the rotating paper at the scanning speed of $\mathrm{v}=360 \mathrm{~mm} / \mathrm{sec}$ are shown in Fig. 9 for different insertion lengths lp $(\mu \mathrm{m})$. $\mathrm{t}$ is recognized from the SEM images Fig. 9 (a) ( f) that the amount of the abraded damage on the silicone rubber sheet becomes larger as the applied pressure of the rotating paper becomes stronger. Correlation between the insertion length $\mathrm{lp}$ of the rotating paper ( $\mathrm{v}$ $=360 \mathrm{~mm} / \mathrm{sec}$ ) cut by scissors and the abraded damage depth ld of the silicone rubber is shown in Fig. 10. The starting point or the threshold of the abraded damage was estimated $\mathrm{lp} \simeq 70 \mu \mathrm{m}$ using the liner approximation for the plots in the figure.

In the case of the paper cut by scissors, the abraded damage occurs at the insertion length $l p \simeq 70 \mu \mathrm{m}$ which is considerably smaller than the length of the paper cut by the $\mathrm{CO}_{2}$ laser (damage threshold $\mathrm{lp} \simeq 115 \mu \mathrm{m}$ ). The coefficient of increase of the damage enhances by about $30 \%$ compared with that of the laser processed paper. Especially, in the case of over $1 \mathrm{p}=200 \mu \mathrm{m}$, almost two times larger depth of abraded damage was observed.

As a result, the paper cut by a $\mathrm{CO}_{2}$ laser at the optimum condition is estimated to be gentler for the human skin compared with the paper cut by scissors (cutting tool).

\section{Conclusions}

Two effects are mainly examined by using a $\mathrm{CO}_{2}$ laser (lasing wavelength; $10.6 \mu \mathrm{m}$ ) which is possible to cut the paper without contact instead of using a mechanical cutter (slitter).

(1) The blade of the slitter becomes wear after cutting many papers, and the routine replacement of the slitter is required. We examined using a SEM that good cut edges are obtained for the moving paper without burn marks by optimizing the irradiation condition of the $\mathrm{CO}_{2}$ laser. The threshold values were derived for the moving speed of the paper or the scanning speed of the laser beam, and 


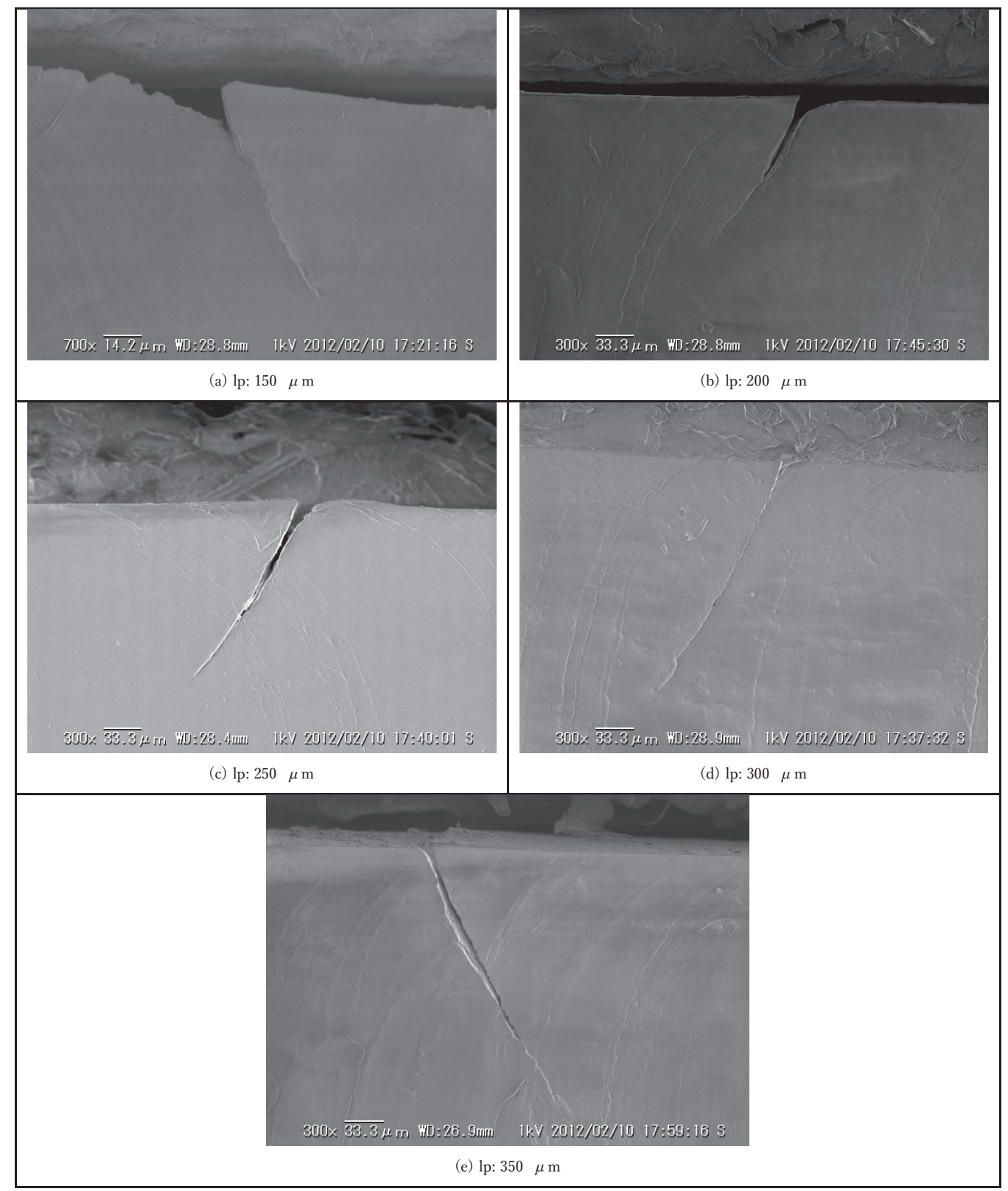

Fig. 7 Cross sectional SEM images of abraded damages on silicone rubbers caused by pressing the edge of $\mathrm{CO}_{2}$ laser cut rotating paper $(\mathrm{v}=360 \mathrm{~mm} / \mathrm{sec})$ at different insertion lengths lp $(\mu \mathrm{m})$

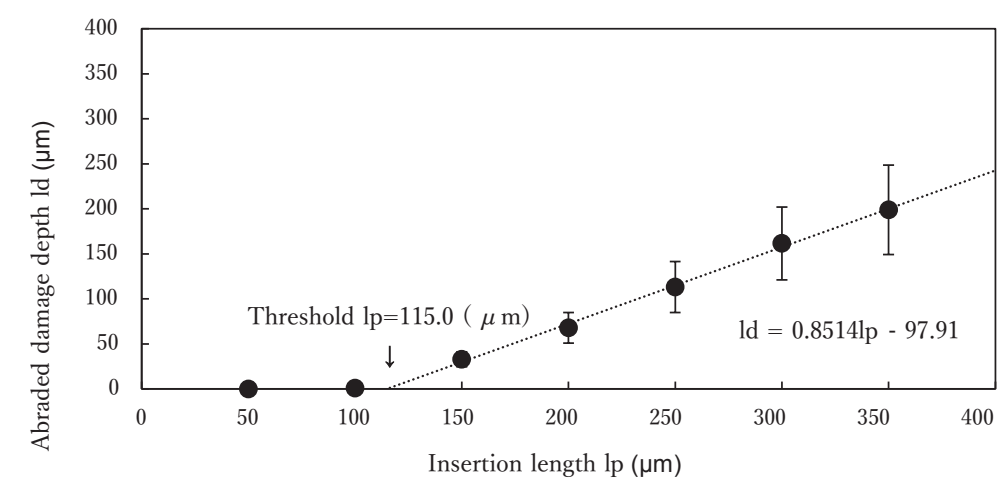

Fig. 8 Correlation between insertion length lp of the rotating paper $(\mathrm{v}=360 \mathrm{~mm} / \mathrm{sec})$ cut by $\mathrm{CO}_{2}$ laser and abraded damage depth ld on silicone rubber sheet 


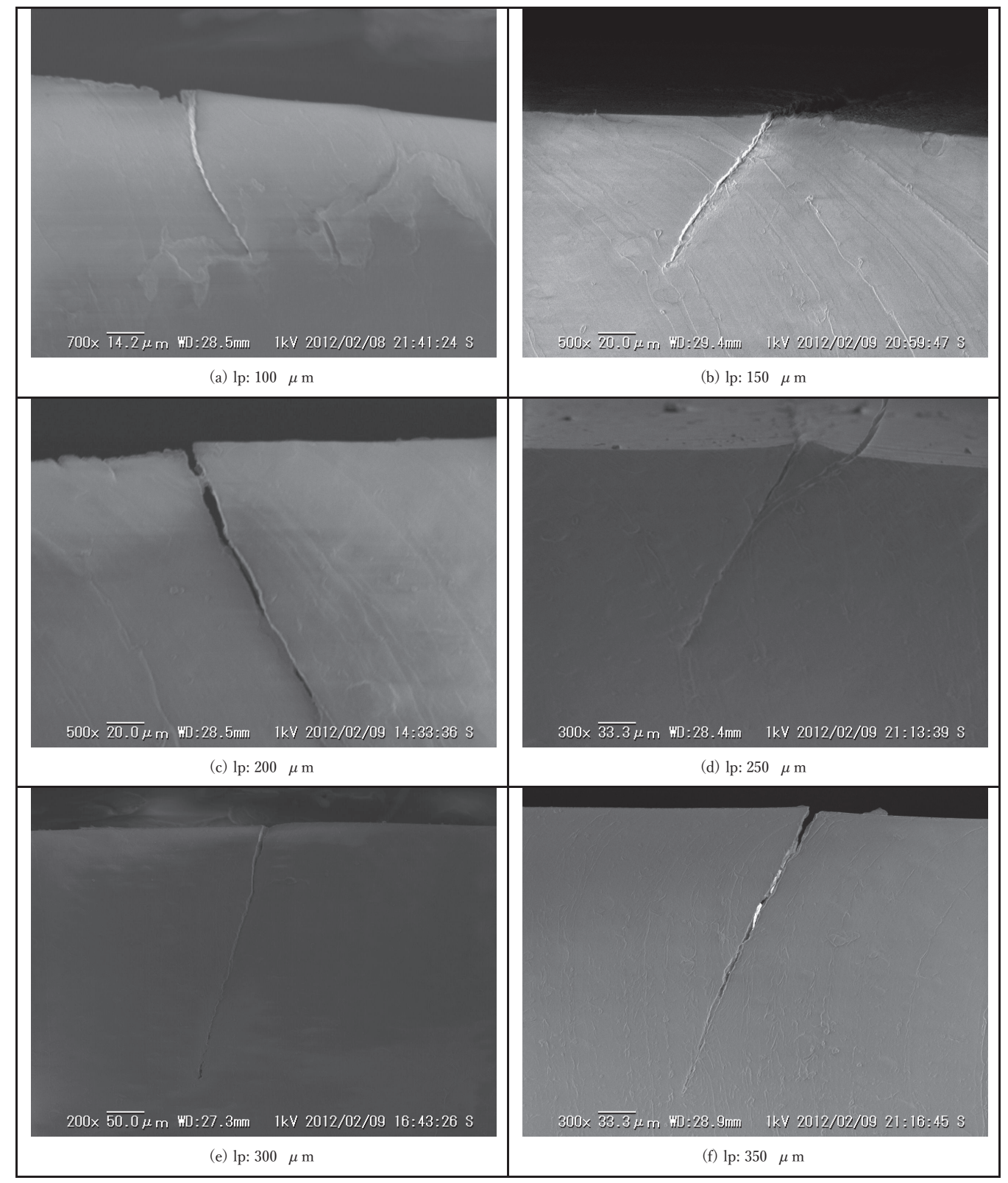

Fig. 9 Cross sectional SEM images of abraded damages on silicone rubbers caused by pressing the edge of scissors (cutting tool) cut rotating paper $(\mathrm{v}=360 \mathrm{~mm} / \mathrm{sec})$ at different insertion lengths $\mathrm{lp}(\mu \mathrm{m})$

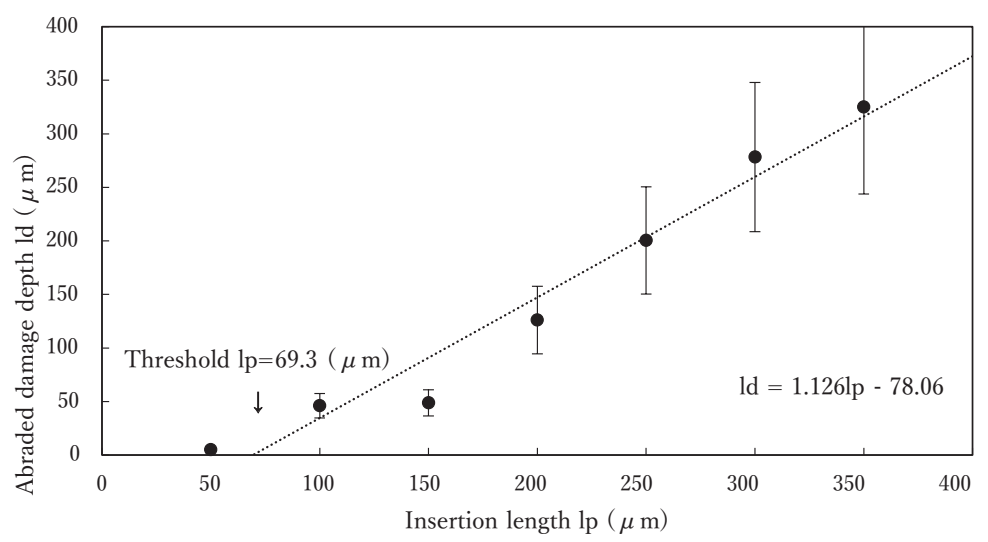

Fig. 10 Correlation between insertion length $\mathrm{lp}$ of the rotating paper $(\mathrm{v}=360 \mathrm{~mm} / \mathrm{sec})$ cut by scissors (cutting tool) and abraded damage depth ld on silicone rubber sheet 
the irradiation laser power density on cutting various copy papers.

(2) We examined the optimum condition in detail to obtain the edge of the paper which offer a gentle edge for human skins with little damage by the abrasion of the edge of the commercial copy paper, especially well used PPC paper (HQ-500). Abrading damages occurred by pressing the moving paper with an edge cut by a laser or a mechanical cutter onto a silicone rubber sheet with the similar property to a human skin at different moving speeds and pressures were observed by a SEM. As for the pressing strength to induce the abrading damage on the silicone rubber sheet, the paper cut by a laser did not induce the abrading damage up to the larger pressure. Even in the damaged region, shallower damage depths which were smaller by $2 / 3$ than that by the paper edge cut by a mechanical cutter was obtained.

As a result, the edge of the paper cut by a laser with the optimum laser parameters is supposed to be safer to human skin than the edge of the paper cut by a cutter. Hereafter, it is requested to examine the effect using a human skin.

\section{Acknowledgments}

We express great thanks to Nobuaki Soujima, Kazuteru Shimada and Masato Suenobu for their cooperation in carrying out various experiments. We also express sincere thanks to KIMMON KOHA Co., Ltd. for the use of $\mathrm{CO}_{2}$ laser.

\section{References}

1) F. Inaba : Introduction to laser engineering (in Japanese), SANBI PRINTING Co., Ltd. 1997, 115-121

2) S. Nemoto: Laser engineering (in Japanese), BAIFUKAN, 2001, 111-114

3) T. Arai : Fundamental Engineering Science for Laser Materials Processing (in Japanese), Maruzen Publishing, 2007, 31-58, 123-127, 141-144, 249-315

4) N. Hirai : Practical laser technology (in Japanese), KYOURITU SHUPPAN, 1987, 160-167

5) N. Hattori, H. Sugihara and Y. Nagano : Zairyou (Materials), Journal of The Society of Materials Science, Japan (in Japanese) 28 (310) 603 (1979)

6) T. Sigeta (THINK LABORATORY) : Patent publication number 10-305380

7) H. Fujitani and M. Funaki (Shoei Printing Co., Ltd.) : Patent publication number 2006-15661

8) Y. Tada, K. Hosoda and M. Asada: Journal of the Robotics Society of Japan 23 (4) 482 (2005) (in Japanese)

9) A. Yariv : Optical Electronics in Modern Communications - Fundamentals (in Japanese), Maruzen Publishing, 2002, 56-60

10) G. Tin and M. Yamamoto : Light and Laser (in Japanese), Ohmsha, 2006, 131-133 